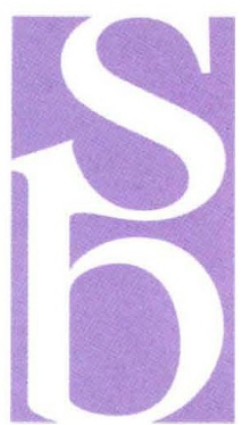

\section{Modelling, folding, kinks and constrictions}

Interest in membrane proteins is very much on the rise as papers in this issue of Nature Structure Biology, on the modelling of the pore protein phospholamban ${ }^{1}$ and the folding of bacteriorhodopsin ${ }^{2}$, and in a recent issue of Nature on the structure of the activated acetylcholine receptor ${ }^{3}$, demonstrate. The cell membrane insulates the cell from the vagaries of the surrounding environment, providing a biochemically isolated compartment in which the reactions that sustain life can be carried out, far from thermodynamic equilibrium. But its isolation cannot be complete. Interaction with the environment is vital and for the most part is mediated by proteins residing in the membrane. These proteins are clearly of the utmost importance to the biochemistry of the cell, the difficulty of analysing them by conventional techniques, in isolation let alone in the context of the lipid bilayer, means that relatively little is know about the details of their structure and function. There is now a prospect that things are changing however, as new methodologies are being brought to bear on the problem.

Brünger and colleagues ${ }^{1}$ describe a method for molecular modelling of multimeric transmembrane helical bundles, a common pattern for the construction of transmembrane channels. They have chosen to model the structure of phospholamban (PLN), a small 52 amino acid protein integral to the membrane of the sarcoplasmic reticulum. As well as being involved in the regulation of $\mathrm{Ca}^{2+}$ ATPase, phospholamban is also a passive ionchannel of unknown function. The pore itself is pentameric and the influence of random mutations in the hydrophobic carboxy terminus on pentamer stability has been explored in earlier studies at Brünger's labora- tory. Now the group has used that earlier data together with molecular modelling of the last $18 \mathrm{C}$-terminal amino acids (which are likely to form the transmembrane region) to determine the most probable structures of the PLN pore.

Consideration of all possible helix-helix interactions in multimeric helical transmembrane proteins would be computationally very time-consuming. A significant simplification of the modelling process is achieved by considering only the interaction between pairs of neighbouring helices in the pentameric transmembrane region of PLN. For a complete conformational search of a heteromeric transmembrane protein, the computational costs of the modelling will increase as the power of the number of helices; analysing a series of two-body interactions reduces considerably the complexity of the analysis (althought in the case of PLN, where the subunits are identical, the complete search method is no more difficult than the two-body search).

Both the two-body search and the fivefold symmetric search converge on a small collection of similar pentameric structures for PLN. Of the most energetically stable structures only one accords well with the mutagenesis data and is found not to be the structure selected by using lowest energy as a criterion. Thus while the computational search methods are able to identify a small number of chemically reasonable models, selection of the most plausible structure benefits immensely from the availability of experimental (mutagenesis) data on the protein in question.

By contrast with PLN, the acetylcholine receptor $(\mathrm{AChR})$ is a neurotransmitter-gated ion channel which is far from passive in its channelling of ions across the post-synaptic membrane of neurons. The structure of the 
open form of the channel, determined by electron microscopy ${ }^{3}$, provides a glimpse of how binding of ACh to the extracellular domain of the receptor opens the gated pore. There are five subunits, $\alpha \alpha \beta \gamma \delta$, arranged in a ring. The central channel provides the pathway for passage of the ions through the membrane. In the absence of ACh the channel is constricted (and presumably blocked) at the level of the membrane where five $\alpha$-helices (the M2 segment from each subunit), come together. In the continuous presence of $\mathrm{ACh}$, the receptor, rather than staying open, undergoes rapid desensitization and closes.

To prepare the open receptor, Unwin has used an ingenious but simple time-of-flight technique to achieve ACh-receptor reaction times of less than $5 \mathrm{~ms}$, thereby minimizing desensitization. A solid support coated with $\mathrm{AChR}$ is dropped into liquid nitrogen-cooled ethane: just before it hits the surface of the ethane the support is sprayed with neurotransmitter. The structure of the frozen receptor-ACh complexes are then analysed by electron microscopy.

In the activated receptor structure the $\mathrm{ACh}$ is thought to bind in ACh 'binding cavities' on the two $\alpha$-subunits of the pentameric ring. The rods (helices) that surround the binding sites within the $\alpha$-subunits are perturbed in response to this binding. The twisting of the $\alpha$ subunit rods is propagated down the shaft of the channel to the symmetrical array of M2 helical segments that line the constricted region of the channel at the level of the membrane.

The M2 helices are kinked in both the closed and open AChR structures: it is suggested that the differing orientation of these kinked helices determine whether ions may pass freely through the channel or not. In the closed structure, the constriction is formed where the 'elbows' of the five helices point into the channel. Unwin has suggested that in the closed channel a ring of leucine residues at the level of the kinks associate through their bulky side-chains to form a hydrophobic plug, blocking the pore ${ }^{4}$. The perturbations transmitted down the $\alpha$-subunits in the ACh-bound receptor causes the M2 helices to rotate over to the side of the channel in such a way that their elbows are now pointing tangentially away from the centre of the pore. The ring of blocking leucine residues would then be broken open and the channel freed from obstruction.

The kinked helices in the open structure are inclined relative to the axis of the channel so that their closest approach is now at the bottom of their length (on the cytoplasmic side of the membrane). Assuming ideal helicity for the M2 segments, Unwin is able to place a threonine reside at the point of closest approach, substitutions of which have been shown to be critical in restricting or enhancing ion flow depending on the volume of the substituted side chain. The events surrounding the binding of $\mathrm{ACh}$ provides an intriguing glimpse of the mechanism of ion channel function. The challenge must now be to fill in the details of the process.

How do PLN, AChR and other membrane proteins fold in the membrane? The scarcity of data on transmembrane protein folding will be alleviated somewhat by the application of stopped flow kinetic measurements of membrane protein refolding in micells, as tackled by Booth and colleagues ${ }^{2}$. Using fast mixing techniques, early kinetic intermediates are identified on the refolding pathway of bacteriorhodopsin. The authors present what must clearly be a tentative refolding pathway for bacteriorhodopsin, given the potential complexity of the process and the difficulty in following the reaction in micelles. Indeed, structural details of the intermediates remain very much a mystery. Nonetheless, the techniques and results described should encourage researchers trying to understand membrane protein folding.

1. Adams, P.D., Arkin, I.T., Engleman, D.M. \& Brunger, A.T. Nature struct. Biol. 2, 154-162 (1995).

2. Booth, P.J. et al. Nature struct. Biology 2 139-143 (1995).

3. Unwin, N. Nature 373, 37-43 (1995).

4. Unwin, N. J. molec Biol. 229, 11011124 (1995).

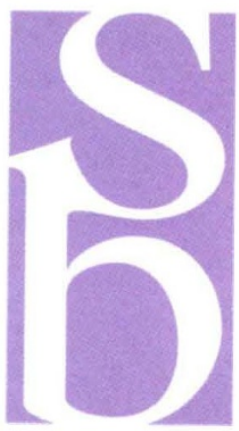

